\title{
Analysis of bilirubin UDP-glucuronosyltransferase gene mutations in an unusual Crigler-Najjar syndrome patient
}

\author{
WEI-LIANG LIU ${ }^{1}$, FANG LI ${ }^{2}$, ZHI-XU HE ${ }^{1}$, HONG-YU JIANG ${ }^{1}$, \\ RONG AI $^{1}$, XIAO-XIA CHEN ${ }^{1}$ and KANG HUANG ${ }^{1}$
}

Departments of ${ }^{1}$ Pediatrics, and ${ }^{2}$ Ophthalmology, Affiliated Hospital of Guiyang Medical College, Guiyang 550004, P.R. China

Received February 2, 2012; Accepted June 8, 2012

DOI: $10.3892 / \mathrm{mmr} .2012 .950$

\begin{abstract}
Crigler-Najjar (CN) syndrome is a rare autosomal recessive inherited disorder characterized by non-hemolytic, unconjugated hyperbilirubinemia. The levels of serum bilirubin and the response to phenobarbital treatment have been used to classify $\mathrm{CN}$ syndrome into two types: $\mathrm{CN}$ I and II. Mutations of the UGTIAl gene have been found to be responsible for cases of $\mathrm{CN}$ syndrome. In the present study, the clinical features of a boy with an unusual type of $\mathrm{CN}$ syndrome were analysed. A DNA sample was obtained from the patient, and the promoter region, the exons and flanking intronic sequences of the UGT1Al gene were analysed using the polymerase chain reaction and sequencing. The case was similar to $\mathrm{CN}$ type I in clinical features, but the therapeutic efficacy in the patient was superior to that typically observed in CN type II disease. Sequencing revealed compound heterozygous mutations, c.211G >A (p.G71R), c.1470C >T (p.D490D) and a normal homozygous A[TA]6TAA. No similar case has been reported worldwide and, considering the specific clinical features and therapeutic efficacy, a distinct type of $\mathrm{CN}$ was suspected. The phenotype of this unusual $\mathrm{CN}$ syndrome patient may be associated with the specific genotype.
\end{abstract}

\section{Introduction}

Crigler-Najjar $(\mathrm{CN})$ syndrome is a rare autosomal recessive inherited disorder characterized by non-hemolytic, unconjugated hyperbilirubinemia (1). The disease is caused by defective activity of the hepatic enzyme bilirubin uridine 5'-diphosphate (UDP)-glucuronosyltransferase. The levels of serum bilirubin and the response to phenobarbital treatment have been used to classify $\mathrm{CN}$ syndrome into two types: CN I and II. CN type I is characterized by severe unconjugated hyperbilirubinemia

Correspondence to: Dr Wei-Liang Liu and Dr Zhi-Xu He, Department of Pediatrics, Affiliated Hospital of Guiyang Medical College, Guiyang 550004, P.R. China

E-mail: liuweiliang205@yahoo.com.cn

E-mail: hezx306@yahoo.com.cn

Key words: Crigler-Najjar syndrome, hyperbilirubinemia, bilirubin UDP-glucuronosyltransferase gene, synonymous mutation, compound heterozygous
( $\geq 340 \mu \mathrm{mol} / \mathrm{l}$ ) from birth, owing to the absence of bilirubinUDP-glucuronosyltransferase (UGT) activity. CN I patients are refractory to phenobarbital administration. Unless treated with liver transplantation, $\mathrm{CN}$ I is lethal during the neonatal period or in infancy. $\mathrm{CN}$ type II occurs due to a severe, but incomplete deficiency of UGT hepatic activity. It is characterized by chronic unconjugated hyperbilirubinemia which is partially responsive to phenobarbital induction (2). Most of the UGT enzymatic activity results from the expression of the UGT1Al gene. Mutations of the UGT1Al gene have been found to be responsible for cases of $\mathrm{CN}$ syndrome $(3,4)$.

In the present study, we investigated an unusual $\mathrm{CN}$ syndrome patient with extremely high levels of unconjugated hyperbilirubin. The patient recovered well from the illness when treated with phototherapy and phenobarbital, and a molecular analysis of the UGTIAl gene was performed.

\section{Case report}

The patient was a 4-month-old Chinese boy. He was born at term (weight 3,500 g) by normal vaginal delivery following a pregnancy of normal duration. The patient was $\mathrm{O}$ Rhesuspositive and was breast-fed. The family noted his icterus when he was 3 days old. He developed severe jaundice and suffered from kernicterus (spasm period). He was first admitted to hospital when he was 7 days old; his serum total and indirect bilirubin concentrations were $686.58 \mu \mathrm{mol} / \mathrm{l}(40.4 \mathrm{mg} / \mathrm{dl})$ and $666.82 \mu \mathrm{mol} / \mathrm{l}(39.2 \mathrm{mg} / \mathrm{dl})$, respectively. He showed no signs of hemolysis, infection or liver dysfunction. The patient was treated with phototherapy ( $66 \mathrm{~h}$ ) for 7 days and phenobarbital ( $5 \mathrm{mg} / \mathrm{kg} /$ day) for 3 days. Following this treatment, his elevated total serum bilirubin levels decreased to $91.8 \mu \mathrm{mol} / \mathrm{l}(5.4 \mathrm{mg} / \mathrm{dl})$ and his psychomotor development recovered to normal. He passed hearing screening in the ears by examination with transiently evoked otoacoustic emission when he was 12 days old. The patient received no treatment after he was 14 days old. At 4 months of age, he had a return visit, during which he exhibited no jaundice or mental or growth retardation. The patient was born to non-consanguineous parents without icteric family history.

Mutation analysis. A blood sample was collected from the patient and genomic DNA was isolated from peripheral blood lymphocytes using standard procedures. All coding exons, 
exon-intron boundaries and the promoter region of UGTIAI were amplified by the polymerase chain reaction (PCR) using standard primers and amplification conditions as described previously $(5,6)$. PCR was performed in a $50-\mu 1$ reaction system containing 10X PCR buffer, $0.1 \mathrm{mmol} / \mathrm{l}$ deoxyribonucleotide triphosphates (dNTPs), $20 \mathrm{mmol} / 1 \mathrm{Mg}^{2+}, 200 \mathrm{ng}$ genomic DNA, $10 \mathrm{pmol} / \mu$ l of each sense and antisense primer and 1.25 units Taq DNA polymerase. The PCR products were subjected to gel electrophoresis for purification and sequencing using an $\mathrm{ABI}$ Prism $3730 \mathrm{XL}^{\circledR}$ sequencer (Applied Biosystems, Carlsbad, CA, USA). The study was performed according to the Declaration of Helsinki and approved by the Ethics Committee of the Affiliated Hospital of Guiyang Medical College. Informed consent was obtained from the patient's parents.

\section{Results and discussion}

The patient affected with $\mathrm{CN}$ syndrome underwent mutation analysis by sequencing of the PCR-amplified DNA fragments, including all coding exons, exon-intron boundaries and promoter region sequences, of the UGTIAl gene. Sequencing revealed compound heterozygous mutations, a missense mutation c.211G $>$ A (p.G71R) in the firstexon (Fig. 1) in which glycine $(\mathrm{G})$ is substituted for arginine (R) at amino acid residue 71 of the UGT, and a synonymous mutation c.1470C $>$ T (p.D490D) in the fifth exon (Fig. 2), which has previously been reported as a rare single-nucleotide polymorphism (SNP) rs114123636, not the substitution of an aspartate (D) at amino acid residue 490. The D490 residue is highly conserved among mammals. Gene analysis also showed a normal homozygous TATA box (A[TA]6TAA) in the promoter element of the patient.

Several authors have analysed genotype-phenotype correlations in $\mathrm{CN}$ syndrome. As previously outlined, chain terminating mutations are more commonly associated with a CN I phenotype, whereas missense changes are more frequently observed among less severely affected CN II patients (7). The serum bilirubin concentration of the patient was consistent with the bilirubin levels of $\mathrm{CN}$ type I, however, the therapeutic efficacy in the patient was superior to that observed in $\mathrm{CN}$ type II disease. In the patient, two genetic lesions coexisted in the UGT1A1 gene, the c.211G $>\mathrm{A}$ and c.1470C $>\mathrm{T}$ mutations. c. $211 \mathrm{G}>\mathrm{A}$ (p.G71R) is the most prevalent mutation in east Asian patients with CN II (8). A single heterozygous G71R missence mutation appears to be insufficient for the severe phenotype in the patient. Taking these findings into consideration, we propose that a synergistic reaction may occur between the c.211G $>\mathrm{A}$ and $\mathrm{c} .1470 \mathrm{C}>\mathrm{T}$ mutations, leading to clearly reduced UGT enzymatic activity. We speculate that the c. $1470 \mathrm{C}>\mathrm{T}$ synonymous mutation in the individual may cause a reversible partial loss of UGT activity. During the translation of mRNA to protein, the c.1470C $>\mathrm{T}$ mutation may lead to a decrease of corresponding tRNA for a period of time, resulting in a loss or a decrease of UGT enzymatic activity. The activity of UGT with the synonymous mutation may increase to normal levels in the later stages of the neonatal period, since the patient returned to normal without any treatment after he was 14 days old. To the best of our knowledge, functional analyses have not been performed to determine the molecular results of the c.1470C $>\mathrm{T}$ synonymous mutation, but the clinical

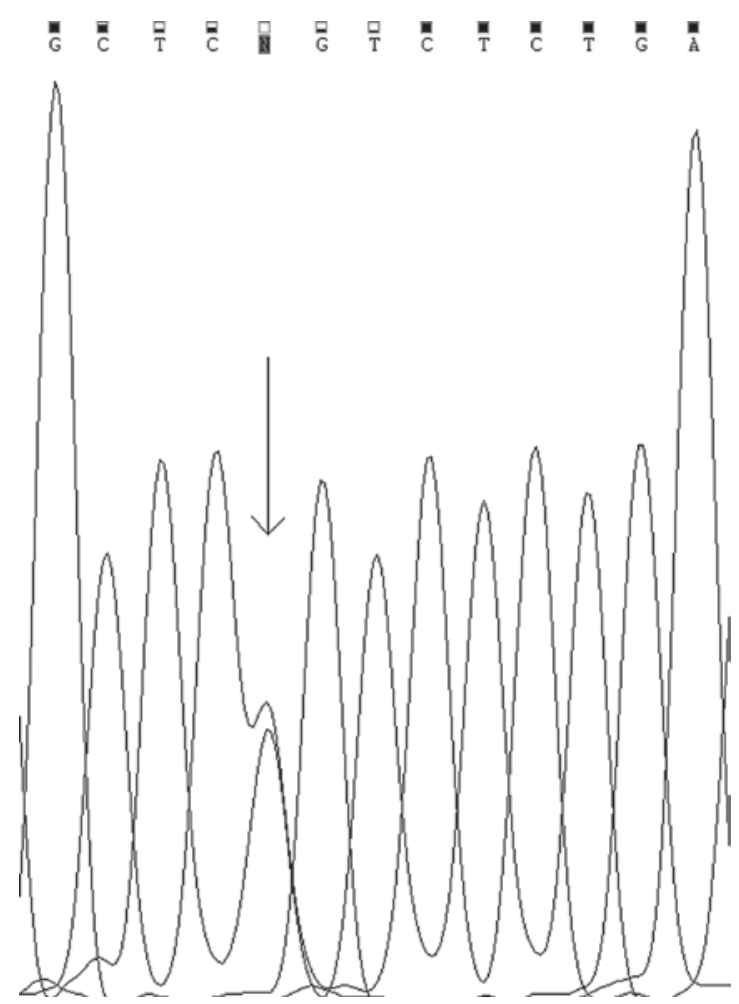

Figure 1. Mutation analysis of exon 1 of the UGT1A1 gene by reverse sequencing, c.211G $>$ A (p.G71R); the mutation is shown by an arrow.

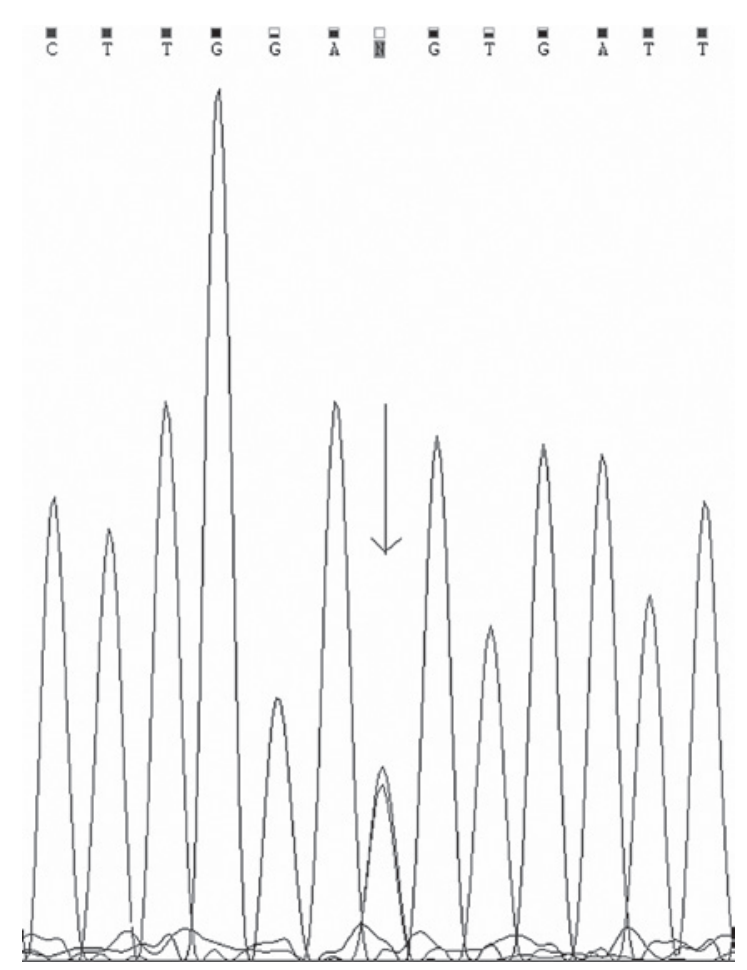

Figure 2. Mutation analysis of exon 5 of the UGT1A1 gene by direct sequencing, c.1470C >T (p.D490D); the mutation is shown by an arrow.

features of this particular $\mathrm{CN}$ syndrome patient indicate that the synonymous mutation is important for enhancing the effect of the heterozygous coding mutation c.211G $>$ A (p.G71R). The phenotype of this unusual $\mathrm{CN}$ syndrome patient may be associated with the specific genotype. 
As previously reported, in order to detect any genotypephenotype correlation in $\mathrm{CN}$ syndrome patients it is mandatory to evaluate the (TA)7 promoter of the UGT1A1 gene (9). Our patient did not have the polymorphism in the promoter region. The present study revealed that the polymorphism of the coding region may affect the expression of the UGTIAl gene. In the present study, we reported that this particular $\mathrm{CN}$ syndrome patient with the genotype defined in this study had clinical features similar to $\mathrm{CN}$ type I, but had a superior response to therapy than is usual for CN type II. No similar case has been reported worldwide. Considering the specific clinical features and therapeutic efficacy, a distinct type of $\mathrm{CN}$ was suspected. The results of this study may be useful in improving the precision of future genotype-phenotype correlation studies.

\section{Acknowledgements}

The authors thank the patient and his family and the clinicians who collaborated in this study. This study was supported by the Section of Scientific Research, Science and Technology, Department of Guizhou Province, China [code (2010)3173] and by the key project of the Chinese Ministry of Education (No. 212155).

\section{References}

1. Crigler JF and Najjar VA: Congenital familial non-hemolytic jaundice with kernicterus. Pediatrics 10: 169-179, 1952.

2. Servedio V, d'Apolito M, Maiorano N, et al: Spectrum of UGT1A1 mutations in Crigler-Najjar (CN) syndrome patients: identification of twelve novel alleles and genotype-phenotype correlation. Hum Mutat 25: 325, 2005.
3. Aono S, Yamada Y, Keino H, et al: Identification of defect in the genes for bilirubin UDP-glucuronosyl-transferase in a patient with Crigler-Najjar syndrome type II. Biochem Biophys Res Commun 197: 1239-1244, 1993.

4. Aono S, Yamada Y, Keino H, et al: A new type of defect in the gene for bilirubinuridine 5'-diphosphate-glucuronosyltransferase in a patient with Crigler-Najjar syndrome type I. Pediatr Res 6: 629-632, 1994

5. Bosma PJ, Roy Chowdhury $\mathrm{N}$, Goldhoorn BG, et al: Sequence of exons and flanking regions of human bilirubin UDP-glucuronosyltransferase gene complex and identification of a genetic mutation in a patient with Crigler-Najjar syndrome type I. Hepatology 15: 941-947, 1992.

6. Ritter JK, Yeatman MT,Ferreira P, et al: Identification of a genetic alteration in the code for bilirubin UDP-glucuronosyltransferase in the UGT1 gene complex of a Crigler-Najjar type I patient. J Clin Invest 9: 150-155, 1992.

7. Kadakol A, Ghosh SS, Sappal BS, et al: Genetic lesions of bilirubin-diphosphoglucuronate glucosyltransferase (UGT1A1) causing Crigler-Najjar Gilbert syndromes: correlation of genotype to phenotype. Hum Mutat 4: 297-306, 2000.

8. Akaba K, Kimura T, Sasaki A, et al: Neonatal hyperbilirubinemia and mutation of the bilirubin uridine diphosphate-glucuronosyltransferase gene: a common missense mutation among Japanese, Koreans and Chinese. Biochem Mol Biol Int 46: 21-26, 1998.

9. Kakadol A, Sappal BS, Ghosh SS, et al: Interaction of coding region mutations and the Gilbert-type promoter abnormality of the UGT1A1 gene causes moderate degrees of unconjugated hyperbilirubinaemia and may lead to neonatal kernicterus. J Med Genet 38: 244-249, 2001 